\title{
THE CHAIN LEMMA FOR KUMMER ELEMENTS OF DEGREE 3
}

\author{
MARKUS ROST
}

\begin{abstract}
Let $A$ be a skew field of degree 3 over a field containing the $3^{\text {rd }}$ roots of unity. We prove a sort of chain equivalence for Kummer elements in $A$. As a consequence one obtains a common slot lemma for presentations of $A$ as a cyclic algebra.
\end{abstract}

\section{Chaînes d'éléments de Kummer en degré 3}

Résumé. Soit $k$ un corps contenant les racines cubiques de l'unité, et soit $A$ un corps gauche de centre $k$, avec $[A: k]=9$. Nous montrons que deux éléments de Kummer de $A$ peuvent être joints par une chaîne de longueur 4.

\section{Version française abrégée}

Soit $k$ un corps contenant une racine primitive $n$-ème de l'unité $\zeta$, et soit $A$ une $k$-algèbre centrale simple de degré $n$. Un élément de Kummer de $A$ est un élément dont le polynôme caractéristique est de la forme $t^{n}-a$, avec $a \in k^{*}$. Par une $\zeta$-paire on entend un couple $(X, Y)$ d'éléments de Kummer de $A$ tels que $Y X=\zeta X Y$. Une telle paire donne une présentation de $A$ comme produit croisé cyclique:

$$
A=\left\langle X, Y \mid X^{n}=a, Y^{n}=b, Y X=\zeta X Y\right\rangle, \quad \text { avec } a, b \in k^{*} .
$$

Soient $X, Y$ deux éléments de Kummer de $A$, et soit $m$ un entier $\geq 1$. Une chaîne de longueur $m$ joignant $X$ à $Y$ est une suite de $m+1$ éléments de Kummer :

$$
X=Z_{0}, Z_{1}, \ldots, Z_{m}=Y
$$

tels que $\left(Z_{i-1}, Z_{i}\right)$ soit une $\zeta$-paire pour $i=1, \ldots, m$.

Supposons que $A$ soit un corps gauche. Si $n=2$ (i.e. si $A$ est un corps de quaternions), il est facile de voir que tout couple d'éléments de Kummer peut être joint par une chaîne de longueur 2 . Si $n=3$, J.-P. Tignol a donné des exemples de couples $(X, Y)$ d'éléments de Kummer tels qu'il n'existe aucune chaîne de longueur 2 joignant $X$ à $Y$ (ni même à un conjugué de $Y$, cf. Appendice); dans ce qui suit, nous montrons qu'un tel couple peut être joint par une chaîne de longueur 4. La démonstration s'inspire de celle donnée par Petersson-Racine [1] pour un résultat analogue dans les algèbres de Jordan exceptionnelles. Comme conséquence, on obtient un "common slot lemma" pour les algèbres de degré 3.

Date: February 19, 1999.

The text appeared as: C. R. Acad. Sci. Paris Sér. I Math. 328 (1999), no. 3, 185-190. 


\section{INTRODUCTION}

The well known common slot lemma for quaternion algebras asserts that if $(a, b)$ is split over $k(\sqrt{c})$, then $(a, b) \simeq(a, e) \simeq(c, e)$ for some $e$.

Till a few years ago not much has been known about similar statements for algebras of degree $>2$. Tignol has given an example (cf. Appendix) which shows that a common slot lemma with just one additional "slot" does not hold in general for algebras of degree 3 . The first positive result was obtained by Petersson and Racine [1] who proved, taking up a suggestion of J-P. Serre, a common slot lemma for exceptional Jordan algebras over quadratically closed fields.

The major purpose of this Note is to present the Petersson-Racine arguments in the much simpler case of central simple algebras of degree 3 . They yield a sort of chain equivalence for Kummer elements. As a consequence one obtains a common slot lemma for such algebras.

I am indebted to Jean-Pierre Tignol for leaving his text on the counterexample as an appendix to this Note.

\section{KumMER ELEMENTS}

Let $n \geq 2$ and let $k$ be a field containing a primitive $n^{\text {th }}$ root of unity $\zeta$. For $a$, $b \in k^{*}$ we denote by $(a, b)$ the $k$-algebra defined by the presentation

$$
\left\langle X, Y \mid X^{n}=a, Y^{n}=b, Y X=\zeta X Y\right\rangle .
$$

Let $A$ be a central simple algebra of degree $n$ over $k$. A Kummer element in $A$ is an element $X \in A$ whose characteristic polynomial $P_{X}$ is of the form $P_{X}(t)=t^{n}-a$ for some $a \in k^{*}$.

Lemma 1.1. Let $X \in A$ be a Kummer element and let

$$
E(X, \zeta)=\{Z \in A \mid Z X=\zeta X Z\} .
$$

(i) $L=k[X]$ is the centralizer of $X$ in $A$.

(ii) There exists $Y \in A^{*}$ such that $Y X Y^{-1}=\zeta X$.

(iii) For $Y$ as in (ii) one has $E(X, \zeta)=Y L=L Y$.

Proof. (i) follows from $\operatorname{dim}_{k} L=\operatorname{deg} A$, (ii) from the Skolem-Noether theorem, and (iii) from (i) and (ii).

By a $\zeta$-pair we understand a pair $(X, Y)$ of invertible elements $X, Y \in A$ such that $Y X=\zeta X Y$.

Lemma 1.2. Let $(X, Y)$ be a $\zeta$-pair.

(i) $X$ and $Y$ are Kummer elements.

(ii) If $A=M_{n}(k)$ and $X^{n}=Y^{n}=1$, then the pair $(X, Y)$ is conjugate to the pair $\left(X_{0}, Y_{0}\right)$, where $X_{0}$ is the diagonal matrix $\operatorname{diag}\left(1, \zeta, \zeta^{2}, \ldots, \zeta^{n-1}\right)$ and where $Y_{0}$ is the permutation matrix $e_{i} \mapsto e_{i-1}$ with $i$ taken $\bmod n$.

(iii) The algebra $A$ has the presentation (*).

Proof. Since $Y X Y^{-1}=\zeta X$, the $n$ different powers of $\zeta$ are roots of $P_{X}$, whence $P_{X}(t)=t^{n}-a$ for some $a \in k$. Further, $X$ is invertible and therefore $a \neq 0$. Similarly one sees $P_{Y}(t)=t^{n}-b$ for some $b \in k^{*}$. This proves (i). For (ii) note that any matrix $X$ with $P_{X}(t)=t^{n}-1$ is conjugate to $X_{0}$ and we may therefore assume $X=X_{0}$. Then necessarily $Y=U Y_{0}$ where $U$ is in the centralizer $L=k[X]$ of $X$. One has $N_{L / k}(U)=Y^{n}=1$. Therefore there exist $V \in L^{*}$ such that 
$U=V Y_{0} V^{-1} Y_{0}^{-1}$. It follows that $V^{-1} Y V=Y_{0}$, which proves the claim. For (iii) one may assume that $k$ is algebraically closed and that $A=M_{n}(k)$. The claim follows from (ii) after replacing $X$ by $X / \sqrt[n]{a}$ and $Y$ by $Y / \sqrt[n]{b}$.

\section{CHAINS}

Let $X, Y \in A$ be Kummer elements. By a chain from $X$ to $Y$ of length $m$ we understand a sequence $X=Z_{0}, Z_{1}, \ldots, Z_{m}=Y$ of Kummer elements in $A$ such that $\left(Z_{i-1}, Z_{i}\right)$ is a $\zeta$-pair for $i=1, \ldots, m$.

Let $Z_{0}, \ldots, Z_{m}$ be a chain of Kummer elements in $A$ and let $a_{i}=Z_{i}^{n}$. Then

$$
A \simeq\left(a_{i-1}, a_{i}\right)
$$

for $i=1, \ldots, m$. This shows that a chain of Kummer elements gives rise to a sequence of presentations $(*)$ with "common slots".

If there exists a chain from $X$ to $Y$ of length $m$, then there exists also a chain from $X$ to $Y$ of length $m^{\prime}$ for any $m^{\prime} \geq m$ (if $X, Y$ is a chain of length 1 , then $X$, $Y X, Y$ is a chain of length 2).

Given Kummer elements $X$ and $Y$, does there exist a chain from $X$ to $Y$ ?

Let us consider the case $n=2$. Then $A$ is a quaternion algebra and $X \in A$ is a Kummer element if and only if $X$ is invertible and trace $(X)=0$. Given Kummer elements $X$ and $Y$, let $Z=X Y-Y X$. If $Z$ is invertible, then $X, Z, Y$ is chain from $X$ to $Y$. If $Z=0$, then $X$ and $Y$ are scalar multiples of each other and any Kummer element $Z^{\prime}$ anti-commuting with $X$ gives rise to a chain $X, Z^{\prime}, Y$. It follows that for quaternion skew fields there exist always chains from $X$ to $Y$ of length 2 . In the case $A=M_{2}(k)$ is not difficult to see that there exist always chains of length 3 and to give examples of Kummer elements $X, Y$ for which there does not exist a chain of length 2 .

We now assume $n=3$.

Proposition 2.1. Let $A$ a skew field of degree 3 over a field containing a primitive $3^{\text {rd }}$ root of unity $\zeta$. Then for any two Kummer elements $X, Y \in A$ there exists a chain of length 4 from $X$ to $Y$.

As an immediate consequence of the proposition one obtains:

Corollary 2.2. Suppose that $(a, b)$ is split over $k(\sqrt[3]{c})$. Then there exist $e, f$, $g \in k^{*}$ such that

$$
(a, b) \simeq(a, e) \simeq(f, e) \simeq(f, g) \simeq(c, g) .
$$

Proof. Let $A=(a, b)$. If $A$ is split, one takes $e=f=g=1$. Assume that $A$ is a skew field and choose Kummer elements $X, Y \in A$ with $X^{3}=a$ and $Y^{3}=c$. By Proposition 2.1 there exists a chain $X, Z_{1}, Z_{2}, Z_{3}, Y$. It suffices to take $e=Z_{1}^{3}$, $f=Z_{2}^{-3}$, and $g=Z_{3}^{-3}$.

Tignol's example in the appendix shows that there exist an algebra $A$ of degree 3 and Kummer elements $X, Y \in A$ for which there is no chain of length 2 from $X$ to any conjugate of $Y$. The question for chains of length 3 is more delicate: it turns out that for generic $X, Y$ there exist exactly 2 chains of length 3 which however might be defined only over a quadratic extension of the ground field. We hope to provide details for this at another occasion. 


\section{Proof of Proposition 2.1}

Let $k$ be a field with char $k \neq 3$ containing a primitive $3^{\text {rd }}$ root of unity $\zeta$. Moreover let $A$ be a skew field of degree 3 and let $X, Y \in A$ be Kummer elements. Let $L=k[X] \subset A$ be the subfield generated by $X$. Then

$$
A=L \oplus E(X, \zeta) \oplus E\left(X, \zeta^{2}\right) .
$$

We show that there exist invertible elements $Z_{1}, Z_{2}, Z_{3} \in A$ such that:

(1) $Z_{1} \in E(X, \zeta)$

(2) $Z_{2} Z_{1}=\zeta Z_{1} Z_{2}$,

(3) $Z_{3} Z_{2}=\zeta Z_{2} Z_{3}$

(4) $Z_{3} \in E\left(Y, \zeta^{2}\right)$

(5) $Z_{2} \in X^{2} k \oplus E\left(X, \zeta^{2}\right)$,

(6) $Z_{3} \in E(X, \zeta) \oplus E\left(X, \zeta^{2}\right)$.

Conditions (1)-(4) mean that $X, Z_{1}, Z_{2}, Z_{3}, Y$ is a chain. The additional conditions (5) and (6) are taken from [1]. Their significance lies in the fact that for generic $X, Y$ the system of equations (1)-(6) has a solution $\left(Z_{1}, Z_{2}, Z_{3}\right), Z_{i} \neq 0$ which is unique up to scalar factors of the $Z_{i}$. It would be interesting to understand more about the geometry of the system (1)-(6). In the following we merely present a solution.

Lemma 3.1. There exist $Z_{3} \neq 0$ satisfying (4) and (6).

Proof. One has $\operatorname{dim}_{k} E\left(Y, \zeta^{2}\right)=3$ and $\operatorname{dim}_{k}\left(E(X, \zeta) \oplus E\left(X, \zeta^{2}\right)\right)=6$. Both vector spaces lie in the 8 -dimensional vector subspace of $A$ of trace zero elements. Hence they have a nontrivial intersection.

We choose $Z_{3}$ as in Lemma 3.1. It remains to find $Z_{1}, Z_{2} \in A^{*}$ satisfying (1), $(2),(3)$, and $(5)$.

Let $Z \in E(X, \zeta), Z \neq 0$. Then $E(X, \zeta)=Z L$ and $E\left(X, \zeta^{2}\right)=L Z^{-1}$. Write

$$
Z_{3}=Z \mu^{\prime}+\mu^{\prime \prime} Z^{-1}
$$

with $\mu^{\prime}, \mu^{\prime \prime} \in L$.

If $\mu^{\prime}=0$, then $Z_{3} \in E\left(X, \zeta^{2}\right)$ and $Z_{1}=Z_{3}^{-1}, Z_{2}=X^{2}$ do the job.

If $\mu^{\prime \prime}=0$, then $Z_{3} \in E(X, \zeta)$ and $Z_{1}=Z_{3} X, Z_{2}=Z_{3}^{2} X$ do the job.

Assume that $\mu^{\prime} \neq 0$ and $\mu^{\prime \prime} \neq 0$. After replacing $Z$ by $Z \mu^{\prime \prime}$ we have $Z_{3}=$ $Z \mu+Z^{-1}$ for some nonzero $\mu \in L$.

Lemma 3.2. Let $(X, Z)$ be a $\zeta$-pair, let $\mu=m_{0}+m_{1} X+m_{2} X^{2}, m_{i} \in k$, and let $T=Z \mu+Z^{-1}$. Let further $c_{2}$ be the second coefficient of the characteristic polynomial of $T$. Then $c_{2}=-3 m_{0}$.

Proof. One has trace $(T)=0$ and $\operatorname{trace}\left(T^{2}\right)=2 \operatorname{trace}(\mu)=6 m_{0}$. Since $2 c_{2}=$ $\operatorname{trace}(T)^{2}-\operatorname{trace}\left(T^{2}\right)$, it follows that $2 c_{2}=-6 m_{0}$. This proves the claim for char $k \neq 2$. For char $k=2$, consider $c_{2}=-3 m_{0}$ as a polynomial identity in the variables $m_{i}$. It suffices to verify this identity for a standard $\zeta$-pair $(X, Z)$ in $M_{3}(\mathbb{Z}[\zeta])$. This follows from the characteristic 0 case.

For the Kummer element $T=Z_{3}$ one has $c_{2}=0$ and Lemma 3.2 shows that

$$
\mu=m_{1} X+m_{2} X^{2}, \quad Z_{3}=Z\left(m_{1} X+m_{2} X^{2}\right)+Z^{-1}
$$

for some $m_{1}, m_{2} \in k$.

If $m_{1}=0$, then $Z_{1}=Z$ and $Z_{2}=(Z X)^{-1}$ do the job. 
Otherwise let

$$
\begin{gathered}
b=Z^{-3}, \quad c=\zeta m_{1} b / N_{L / k}(\mu), \quad \lambda=c \mu X, \\
Z_{1}=Z \lambda, \quad Z_{2}=X^{2}\left(1+(Z \lambda)^{-1}\right) .
\end{gathered}
$$

With these settings, (1), (2), and (5) are obvious. It remains to verify (3):

$$
\left(Z \mu+Z^{-1}\right) X^{2}\left(1+(Z \lambda)^{-1}\right)=\zeta X^{2}\left(1+(Z \lambda)^{-1}\right)\left(Z \mu+Z^{-1}\right) .
$$

To check this, one considers the components with respect to the decomposition $(* *)$. For the first component one gets $Z \mu X^{2} \lambda^{-1} Z^{-1}=\zeta X^{2} \lambda^{-1} Z^{-1} Z \mu$, which follows from $\mu X^{2} \lambda^{-1}=X c^{-1}$ and $Z X=\zeta X Z$. For the third component one gets $Z^{-1} X^{2}=\zeta X^{2} Z^{-1}$, which is immediate from $Z X=\zeta X Z$. For the second component one gets

$$
Z \mu X^{2}+Z^{-1} X^{2} \lambda^{-1} Z^{-1}=\zeta X^{2} Z \mu+\zeta X^{2} \lambda^{-1} Z^{-1} Z^{-1}
$$

This is equivalent to both of the following equations:

$$
\begin{aligned}
X^{2} \mu+Z^{-2} X^{2} \lambda^{-1} Z^{-1} & =\zeta^{2} X^{2} \mu+\zeta^{2} X^{2} Z^{-1} \lambda^{-1} Z^{-2} \\
\left(1-\zeta^{2}\right) X^{2} \mu & =\zeta^{2} X^{2} Z^{-1} \lambda^{-1} Z^{-2}-Z^{-2} X^{2} \lambda^{-1} Z^{-1}
\end{aligned}
$$

For the right hand side of the last equation one computes

$$
\begin{aligned}
\text { r. h. s. } & =\zeta^{2} X^{2} Z^{-1}(c \mu X)^{-1} Z^{-2}-\zeta^{2} X^{2} Z^{-2}(c \mu X)^{-1} Z^{-1} \\
& =c^{-1} X Z^{-1} \mu^{-1} Z^{-2}-c^{-1} \zeta X Z^{-2} \mu^{-1} Z^{-1} \\
& =b c^{-1} X\left(Z^{-1} \mu Z\right)^{-1}-b c^{-1} \zeta X\left(Z^{-2} \mu Z^{2}\right)^{-1} .
\end{aligned}
$$

We multiply both sides with the conjugates $Z^{-1} \mu Z$ and $Z^{-2} \mu Z^{2}$ of $\mu$. Then our equation reads as

$$
\begin{aligned}
\left(1-\zeta^{2}\right) X^{2} N_{L / k}(\mu) & =b c^{-1} X\left(Z^{-2} \mu Z^{2}\right)-b c^{-1} \zeta X\left(Z^{-1} \mu Z\right) \\
& =b c^{-1} X\left(m_{1} \zeta X+m_{2} \zeta^{2} X^{2}\right)-b c^{-1} \zeta X\left(m_{1} \zeta^{2} X+m_{2} \zeta X^{2}\right) \\
& =b c^{-1} m_{1} \zeta X^{2}\left(1-\zeta^{2}\right) .
\end{aligned}
$$

The equality is now clear.

\section{Appendix}

With the kind permission of Jean-Pierre Tignol we reproduce here his text on

A "common slot" counterexample in degree 3

Notation: For $a, b$ nonzero elements in a field $F$ containing a primitive cube root of unity $\omega$, the symbol $(a, b)$ denotes the element of the Brauer group of $F$ represented by the $F$-algebra generated by elements $\alpha, \beta$ subject to

$$
\alpha^{3}=a, \quad \beta^{3}=b, \quad \beta \alpha=\omega \alpha \beta .
$$

Let $a_{1}, b_{1}, a_{2} \in F^{\times}$. If there exist $x, y \in F^{\times}$such that

$$
\left(a_{1}, b_{1}\right)=\left(a_{1}, x\right)+\left(a_{1}, y\right), \quad\left(a_{1}, x\right)=-\left(a_{2}, x\right), \quad\left(a_{1}, y\right)=\left(a_{2}, y\right),
$$

then the additivity of symbols yields $\left(a_{1}, b_{1}\right)=\left(a_{2}, x^{-1} y\right)$. However, the next example shows that when $\left(a_{1}, b_{1}\right)$ is split by $F\left(\sqrt[3]{a_{2}}\right)$, there need not exist elements $x, y$ satisfying $(*)$. 
Example: A global field $F$ containing a primitive cube root of unity and elements $a_{1}, b_{1}, a_{2}, b_{2}$ such that $\left(a_{1}, b_{1}\right)=\left(a_{2}, b_{2}\right)$, but no couple of elements $x, y$ satisfying $(*)$. In particular (taking $x=1$ ), the field $F$ does not contain any element $y$ such that

$$
\left(a_{1}, b_{1}\right)=\left(a_{1}, y\right)=\left(a_{2}, y\right)=\left(a_{2}, b_{2}\right)
$$

Let $F=\mathbb{F}_{7}(t)$, where $t$ is an indeterminate, $a_{1}=t$ and $a_{2}=t(1-t)$. Note that $\left(a_{1}, a_{2}\right)=0$. Therefore, for all places $v$ of $F$, the local invariant $\left(a_{1}, a_{2}\right)_{v}$ is trivial. It follows that in the completion $F_{v}$ of $F$ at $v$ we have either $a_{1} \in F_{v}^{\times 3}$ or $a_{1} \equiv a_{2} \bmod$ $F_{v}^{\times 3}$ or $a_{1} \equiv a_{2}^{2} \bmod F_{v}^{\times 3}$ or $a_{2} \in F_{v}^{\times 3}$, since the (generalized) Hilbert symbol $(,)_{v}:\left(F_{v}^{\times} / F_{v}^{\times 3}\right) \times\left(F_{v}^{\times} / F_{v}^{\times 3}\right) \rightarrow \frac{1}{3} \mathbb{Z} / \mathbb{Z}$ is a nondegenerate alternating pairing.

Consider in particular $v_{1}$ the $t$-adic place and $v_{2}$ the $(t+3)$-adic place. Since $a_{1}, a_{2}$ are uniformizing parameters at $v_{1}$, we have $a_{1}, a_{2} \notin F_{v_{1}}^{\times 3}$; but $a_{1} \equiv a_{2} \bmod F_{v_{1}}^{\times 3}$. On the other hand, $a_{1}$ and $a_{2}$ have non-cube residues at $v_{2}$, hence $a_{1}, a_{2} \notin F_{v_{2}}^{\times 3}$ but $a_{1} \equiv a_{2}^{-1} \bmod F_{v_{2}}^{\times 3}$.

Let now $A$ be the central simple $F$-algebra with local invariants $1 / 3$ at $v_{1}, 2 / 3$ at $v_{2}$ and 0 everywhere else. If $v$ is a place of $F$ where $a_{1} \in F_{v}^{\times 3}$, then $v \neq v_{1}, v_{2}$ hence $[A]_{v}=0$. It follows that $A$ is split by $F\left(\sqrt[3]{a_{1}}\right)$, hence we may find $b_{1} \in F^{\times}$ such that $[A]=\left(a_{1}, b_{1}\right)$ in the Brauer group of $F$. Similarly, $A$ is split by $F\left(\sqrt[3]{a_{2}}\right)$ hence we may find $b_{2} \in F^{\times}$such that $[A]=\left(a_{2}, b_{2}\right)$; thus,

$$
\left(a_{1}, b_{1}\right)=\left(a_{2}, b_{2}\right) \text {. }
$$

Suppose now $x, y \in F^{\times}$satisfy $(*)$. Since $a_{1} \equiv a_{2} \bmod F_{v_{1}}^{\times 3}$, the relation $\left(a_{1}, x\right)_{v_{1}}=$ $-\left(a_{2}, x\right)_{v_{1}}$ implies $\left(a_{1}, x\right)_{v_{1}}=0$. On the other hand, since $a_{1} \equiv a_{2}^{-1} \bmod F_{v_{2}}^{\times 3}$, it follows from $\left(a_{1}, y\right)_{v_{2}}=\left(a_{2}, y\right)_{v_{2}}$ that $\left(a_{1}, y\right)_{v_{2}}=0$, hence $\left(a_{1}, x\right)_{v_{2}}=\left(a_{1}, b_{1}\right)_{v_{2}}=$ $2 / 3$.

For $v \neq v_{1}, v_{2}$, we consider four cases, according to the relation between $a_{1}$ and $a_{2}$ in the group of cube classes:

- if $a_{1} \in F_{v}^{\times 3}$, then clearly $\left(a_{1}, x\right)_{v}=0$.

- if $a_{1} \equiv a_{2} \bmod F_{v}^{\times 3}$, then $\left(a_{1}, x\right)_{v}=0$ as for $v=v_{1}$ above.

- if $a_{1} \equiv a_{2}^{-1} \bmod F_{v}^{\times 3}$, then $\left(a_{1}, x\right)_{v}=\left(a_{1}, b_{1}\right)_{v}$ as for $v=v_{2}$ above, hence $\left(a_{1}, x\right)_{v}=0$.

- if $a_{2} \in F_{v}^{\times 3}$, then $\left(a_{1}, x\right)_{v}=0$ follows from $\left(a_{1}, x\right)=\left(a_{2}, x^{-1}\right)$.

Thus, the invariants of $\left(a_{1}, x\right)$ are:

$$
\left(a_{1}, x\right)_{v_{2}}=2 / 3, \quad \text { and } \quad\left(a_{1}, x\right)_{v}=0 \quad \text { for } v \neq v_{2},
$$

a contradiction to the reciprocity law.

Jean-Pierre Tignol, June 1996.

\section{REFERENCES}

[1] H. P. Petersson and M. L. Racine, An elementary approach to the Serre-Rost invariant of Albert algebras, Indag. Mathem., N.S. 7 (1996), no. 3, 343-365.

NWF I - Mathematik, Universität Regensburg, D-93040 Regensburg, Germany

E-mail address: markus.rost@mathematik.uni-regensburg.de

URL: http://www. physik. uni-regensburg. de/ rom03516 